shows that fruit preferences in Asia are different than those in the United States," Crisosto says.

Similar work was carried out with 400 American and 250 native Chinese consumers to determine the two ethnic groups' acceptance of 'Redglobe' table grapes at different maturity levels.

At Kearney, Crisosto conducts fruit tasting tests with panels drawn from staff who have been screened for their taste acuity and trained for a specific test.

"Most of the panelists enjoy it," she says. "They get a break from their work and they know they are making a positive contribution to the furtherance of our scientific knowledge of fruit quality."

Managing fungal diseases. UC Riverside plant pathologist Jim Adaskaveg is leading postharvest pathology research on stone fruit, pome fruit, kiwifruit, pomegranates and citrus. Recently, he initiated a citrus incubation program at the postharvest center that protects the Korean market for the California citrus industry. In 2003, Korea closed its market to California oranges after detecting the fungal disease Septoria spot. After negotiation and study, Adaskaveg and his team, working with the citrus industry and the U.S. Department of Agriculture, arrived at a plan. The team collects and incubates fruit samples at the center, then assesses them 20 days later for Septoria spot. Fruit positive for the disease are identified and photographed, and the results are provided to the packinghouses. This program was in part responsible for maintaining the $\$ 100$ million citrus trade between the two countries.

For other crops, Adaskaveg and his team have developed several new reduced-risk postharvest fungicides. They are evaluating new application strategies to improve disease control (see page 109) and have identified diseases like sour rot that have recently become more of a problem for the stone fruit industry.

Ultimately, all the scientists working on postharvest studies at KREC share the same goal, Gayle Crisosto says. "We want to consistently provide good-tasting fruit to the consumer so they'll come back for more." - Jeannette Warnert

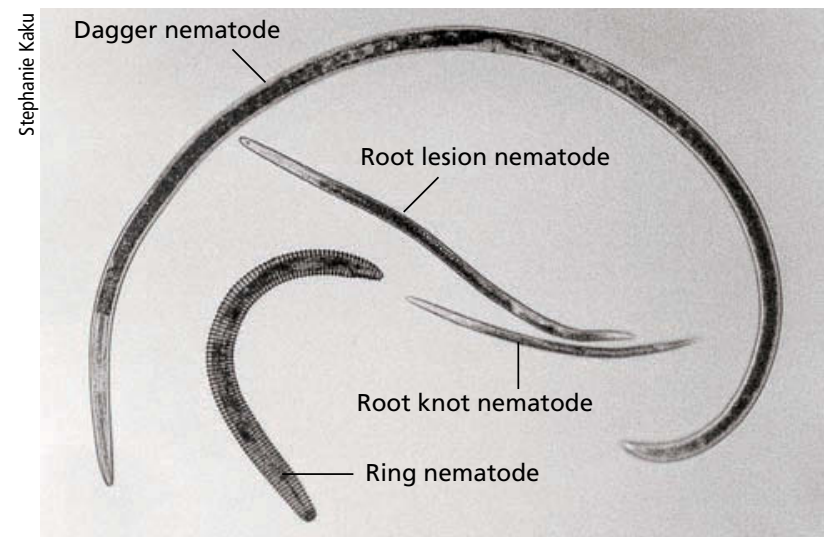

A variety of plant parasitic nematodes can sap nutrients from the roots of trees, vines and field crops.

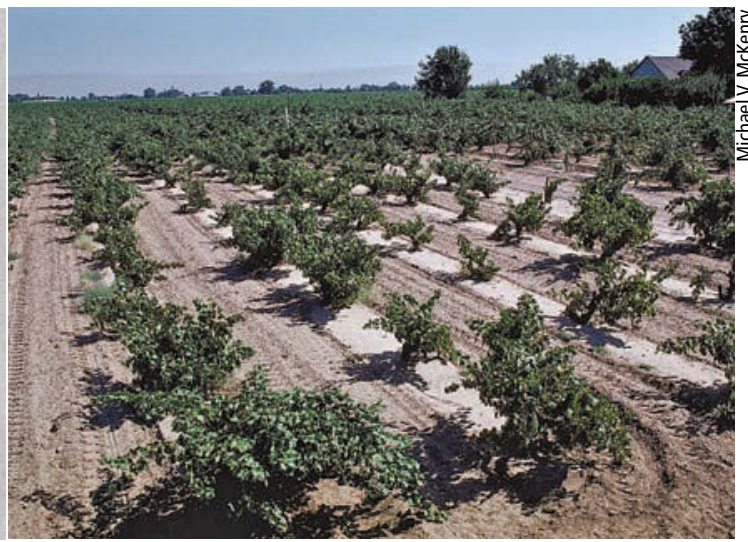

Vines stunted by root knot nematodes.

\section{After years of hosting an orchard or vineyard, nematodes will have colonized and reproduced to levels that would put a new planting in grave danger.}

\title{
UC nematologists battle tiny underground pests
}

$\mathrm{N}$ ematodes are the most numerous multicell animals on earth. One cup of soil can contain thousands of the tiny worms. Some are beneficial, but many cause significant damage to agricultural crops.

"You can see insects, and diseases often cause visible symptoms," says Philip Roberts, a UC Riverside nematologist. "The underground feeding of nematodes can be just as harmful, but it is much more difficult to detect."

Roberts and UC Riverside nematologist Mike McKenry, who is based at the UC Kearney Research and Extension Center, preside over a specialized program that offers pest management professionals and growers the latest information on nematode problems and solutions. Their work is particularly important due to past and upcoming bans on chemicals that have traditionally been used to rid soils of nematodes before planting, such as DBCP and methyl bromide.

Perennial crops. As part of his focus on perennial crops, McKenry has pursued "chemigation" as an alternative to preplant methyl bromide soil fumigations. McKenry has developed methods and equipment that use water to carry low-fuming biocides (with short half-lives), 5 feet deep. Large volumes of water can also prevent biocides from escaping at the field surface. By 1991, he demonstrated that in highly porous soils, chemigation with biocides such as metam sodium could provide nematode control equivalent to that of methyl bromide. "Today, new products and equipment for preplant chemigation are plentiful," McKenry says.

A promising new natural treatment for nematodes 
